\title{
Article \\ Loss of Gene Information: Discrepancies between RNA Sequencing, cDNA Microarray, and qRT-PCR
}

\author{
Nicole Rachinger ${ }^{1}$, Stefan Fischer ${ }^{2}{ }^{\circledR}$, Ines Böhme ${ }^{1}$, Lisa Linck-Paulus ${ }^{1}{ }^{\mathbb{D}}$, Silke Kuphal ${ }^{1}$, \\ Melanie Kappelmann-Fenzl ${ }^{2}$ (D) and Anja K. Bosserhoff ${ }^{1, *(D)}$ \\ 1 Institute of Biochemistry, Friedrich-Alexander University Erlangen-Nürnberg (FAU), Fahrstraße 17, \\ 91054 Erlangen, Germany; nicole.rachinger@fau.de (N.R.); ines.boehme@fau.de (I.B.); \\ lisa.linck@fau.de (L.L.-P.); silke.kuphal@fau.de (S.K.) \\ 2 Faculty of Computer Science, Deggendorf Institute of Technology, Dieter-Görlitz-Platz 1, \\ 94469 Deggendorf, Germany; stefan.fischer@th-deg.de (S.F.); melanie.kappelmann-fenzl@fau.de (M.K.-F.) \\ * Correspondence: anja.bosserhoff@fau.de
}

check for updates

Citation: Rachinger, N.; Fischer, S.; Böhme, I.; Linck-Paulus, L.; Kuphal, S.; Kappelmann-Fenzl, M.; Bosserhoff, A.K. Loss of Gene Information: Discrepancies between RNA Sequencing, cDNA Microarray, and qRT-PCR. Int. J. Mol. Sci. 2021, 22, 9349. https://doi.org/10.3390/ ijms22179349

Academic Editor: Naoyuki Kataoka

Received: 3 August 2021

Accepted: 27 August 2021

Published: 28 August 2021

Publisher's Note: MDPI stays neutral with regard to jurisdictional claims in published maps and institutional affiliations.

Copyright: (c) 2021 by the authors. Licensee MDPI, Basel, Switzerland. This article is an open access article distributed under the terms and conditions of the Creative Commons Attribution (CC BY) license (https:/ / creativecommons.org/licenses/by/ $4.0 /)$.

\begin{abstract}
Molecular analyses of normal and diseased cells give insight into changes in gene expression and help in understanding the background of pathophysiological processes. Years after cDNA microarrays were established in research, RNA sequencing (RNA-seq) became a key method of quantitatively measuring the transcriptome. In this study, we compared the detection of genes by each of the transcriptome analysis methods: cDNA array, quantitative RT-PCR, and RNA-seq. As expected, we found differences in the gene expression profiles of the aforementioned techniques. Here, we present selected genes that exemplarily demonstrate the observed differences and calculations to reveal that a strong RNA secondary structure, as well as sample preparation, can affect RNA-seq. In summary, this study addresses an important issue with a strong impact on gene expression analysis in general. Therefore, we suggest that these findings need to be considered when dealing with data from transcriptome analyses.
\end{abstract}

Keywords: transcriptome analysis; RNA sequencing; cDNA microarray; qRT-PCR; secondary structure; free energy of the RNA secondary structure

\section{Introduction}

To examine the expression of individual genes, quantitative RT-PCR (qRT-PCR) is a widely used method. However, in the past, it became more and more important to analyze the complete transcriptome within cells in an unselected manner for an in-depth understanding of, e.g., development and disease [1]. Starting in 1995, microarrays were used as a standard method for analyzing parts of the transcriptome, until 2008, when the nextgeneration sequencing method, RNA-sequencing (RNA-seq), revolutionized research [2].

DNA microarray analyses, which are based on the hybridization of fluorescently labeled probes, require a reference genome/transcriptome for designing the microarray probes. Therefore, cDNA microarrays are dependent on the choice of probes and their binding specificity. Here, background signals and cross-hybridization can reduce the specificity and sensitivity for some genes, which can lead to pitfalls and misallocations during an analysis [3-7].

Due to these limitations of microarrays and the advantages of RNA-seq, such as cost reduction through massively parallel sequencing, a shift from cDNA array analysis to RNA-seq experiments has been observed in recent years [1,2,8,9]. Nowadays, RNA-seq is a frequently used method with increasing importance for studying differential gene expression, analyzing alternative splicing, and identifying novel RNA species [7,10,11]. Moreover, this technique enables a wider dynamic range for detection and provides less background noise [7]. Despite all of its advantages, RNA-seq is still a technology that is 
in development, and its challenging aspects range from sample preparation (fragmentation method, size selection, library construction) to computational analysis (alignment, quantification, filtering, and normalization), which have a great impact on the overall final results $[10,12]$.

Generally, transcriptome analyses, especially of large sample sets, are usually performed with the newest and most advanced methods. Hardly ever is the same sample set analyzed with both methods in parallel. So far, only a few comparisons that indicate possible discrepancies between the different methods for transcriptome analyses exist $[13,14]$.

In this study, we compared the detection of two groups of genes (the SOX gene family and $Y A P / T A Z$ effectors of the Hippo pathway) in various melanoma cell lines with different transcriptome analysis methods-cDNA array, RNA-seq, and classical quantitative RT$\mathrm{PCR}-$ and revealed striking differences in gene detection. When comparing the expression analysis results using these techniques, we observed that some genes were detectable with qRT-PCR and cDNA arrays, but not with RNA-seq, and vice versa. In this study, we focused on analyzing the loss of information within RNA-seq and, therefore, compared our transcriptome data in different melanoma cell lines with the qRT-PCR and cDNA microarray data, as well as with datasets from different research groups. Our results demonstrate the urgent need for an in-depth understanding regarding RNA-seq data and the related methodological challenges, and we show that there are variances in outcomes with different processing methods.

We herewith suggest that the results of one methodology, which may be state of the art, should always be validated with a second methodology with regard to the experimental setting.

\section{Results and Discussion}

\subsection{Correlation of RNA-Seq and cDNA Microarrays}

In recent years, several groups, such as Fu et al. or Zhao et al., to name a few, developed algorithms for comparing transcriptome data from microarray analyses and RNA-seq $[15,16]$. In this study, however, we do not intend to present an algorithm for the direct comparison of microarray fluorescence signals with read counts from RNA-seq, but rather to point out, in general, that the two methods can reveal discrepancies for the detection of individual genes.

To define the main variations between the results of a cDNA microarray (cDNA array) and RNA-seq, we first analyzed the differences in gene expression obtained by these two methods exemplarily based on melanoma cell lines. We compared the transcriptome analysis results (Supplementary Tables S1 and S2) and performed correlation analyses, which are illustrated as scatter plots (Figure 1). We determined that the gene expression in the melanoma cell line $\mathrm{Mel}$ Im was only detectable with RNA-seq, which was expected due to its methodological advantages with respect to microarray analysis (Figure 1A). Surprisingly, we also found genes that were only detectable with microarray analysis, but not with RNA-seq (Figure 1A, Supplementary Table S3). To demonstrate that this observation is not specific to the cell line, we compared the transcriptome data for another melanoma cell line, Mel Juso (Figure 1B, Supplementary Table S3). Here, there were also genes that were detectable with one method and not with the other transcriptome analyses. In previous studies focusing on the analysis of gene expression with different methods, the loss of gene detection with RNA-seq was not described [13,14]. Due to the unexpected result that genes were not found within the RNA-seq data, but were detectable with the cDNA array analysis, we confirmed our findings by comparing the gene expression observed with microarray or RNA-seq by focusing on individual genes (Figure 1, red label). (The reverse analysis of genes detected with RNA-seq that were not found with the microarray was neglected because of the specific choice of genes analyzed in the microarray.) 
A

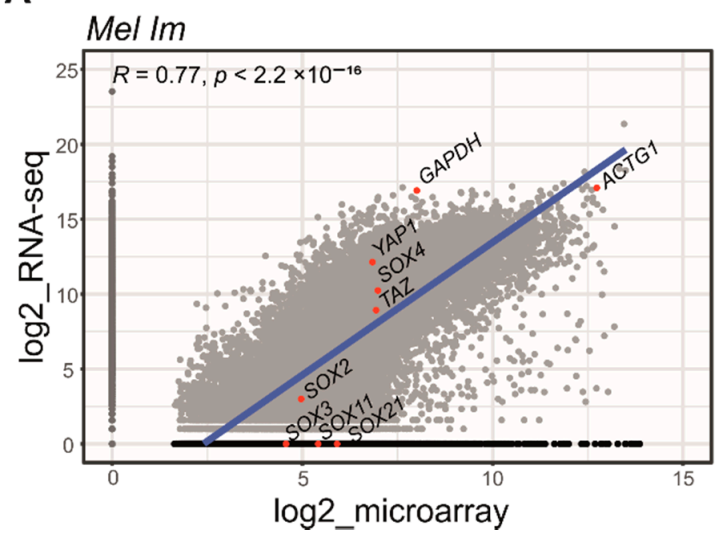

B

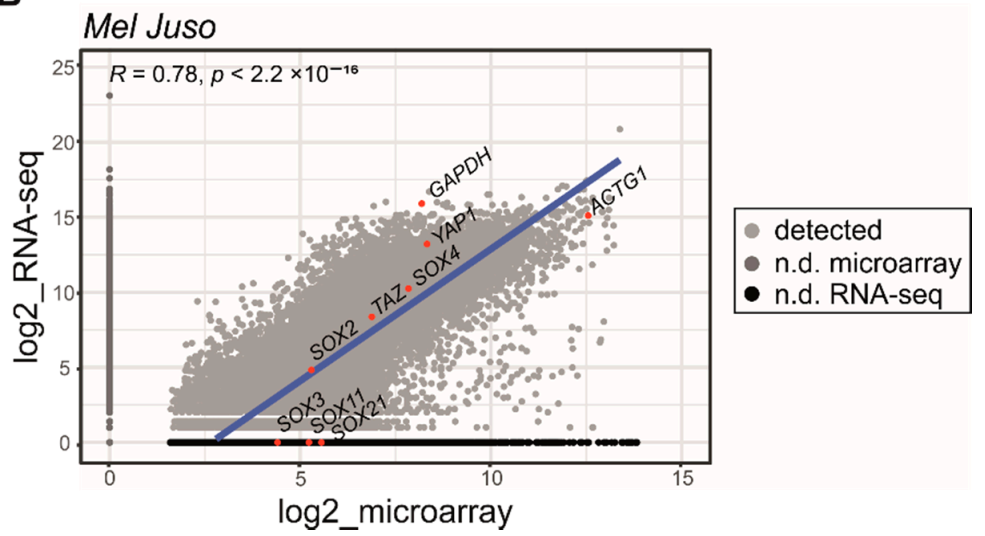

Figure 1. Scatter plots depicting the detected genes measured with two different methods for transcriptome analysis, shown for the melanoma cell line Mel Im in (A) and Mel Juso in (B). The measured signals with log2 are depicted as plots for each gene. The light gray color shows genes that were measured with both methods, the dark gray color shows genes that were not detected (n.d.) with microarray analysis, and the black dots depict genes that were not detected with RNA-seq (genes that were not detected by RNA-seq are listed in Supplementary Table S3). The blue line represents the Spearman correlation between genes that were detected with both methods. The genes named within the plots (labeled in red) are further discussed within the following text. Statistical analysis was performed using R.

\subsection{Analysis of the Loss of SOX Genes with RNA-Seq}

Next, we aimed to identify the possible causes of the observed loss of some of the aforementioned genes (Figure 1A,B, labeled in red). We first analyzed the expression of the housekeepers (glyceraldehyde-3-phosphate dehydrogenase (GAPDH), gamma-actin1 (ACTG1)), which were similarly expressed in each method, cell line, and dataset (GAPDH: Figure 2A; ACTG1: Supplementary Figure S1A). In the following, we focus on the SOX (=SRY-related HMG-box) family members, especially SOX21. We were able to detect SOX21 with the cDNA array; however, we observed no read counts with RNA-seq (Figure 2A, Supplementary Tables S4 and S5). Additionally, we showed these results for further cell lines to demonstrate their independence of the melanoma cell line and consistency across different array and RNA-seq datasets (Supplementary Figure S1A; Supplementary Tables S1, S2, S4 and S5). To underline the expression of SOX21 within the melanoma cell lines and support the microarray data, we analyzed SOX21 on the mRNA (qRT-PCR) (Figure 2B) and protein (Western blot) levels (Figure 2C) and, therefore, confirmed its expression in melanoma cell lines.

Further, we performed qRT-PCR analyses to determine whether the loss of SOX21 in RNA-seq data was due to the library preparation, the mapping algorithm (STAR alignment software v2.5.2a, [17]), or the sequencing process [17]. We used samples produced from RNA with a standard cDNA preparation protocol and compared them with samples used for RNA-seq analysis after enrichment through PCR and ligation of the adapters ("library" or "library sample"; detailed description: Methods and Materials-Analysis of Gene Expression with Quantitative Real-Time PCR; Figure 2D). Corresponding to the RNA-seq data and in contrast to CDNA, the qRT-PCR quantification of the prepared library samples showed no results for SOX21 for the exemplarily used cell lines, indicating that SOX21 was lost during the sample preparation for RNA-seq. To confirm this observation, we searched for two specific SOX21 patterns (TACATGATCCCGTGCAACTG, TTAACCTTTATGTGTAAATG) in the raw RNA-seq fastq files, allowing up to three mismatches. The SOX21 pattern search yielded no hits with up to one mismatch. Random hits started to accumulate with two or more mismatches. A GAPDH pattern served as a positive control and yielded numerous hits with no mismatches. Thus, we can exclude that the detected loss of SOX21 read counts was a mapping artifact (Figure 2E). 
A

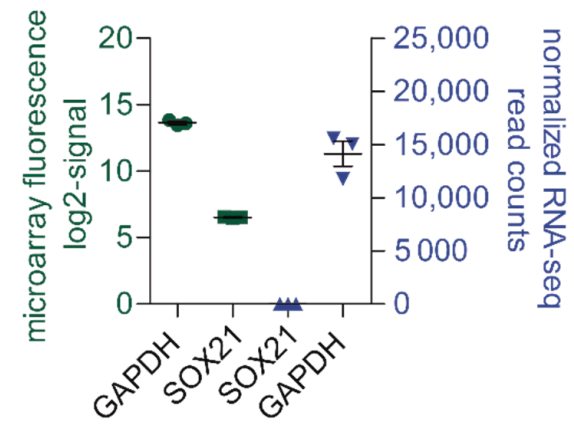

D

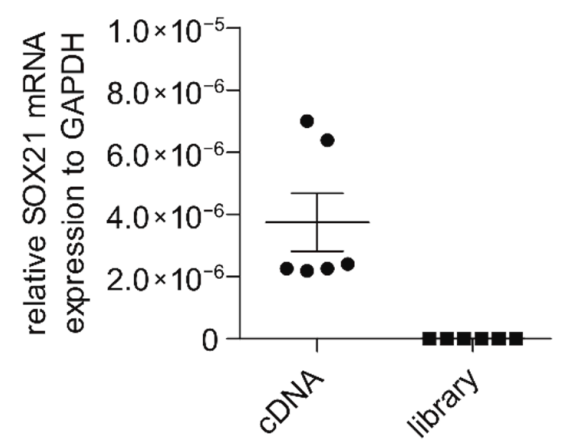

$\mathbf{F}$

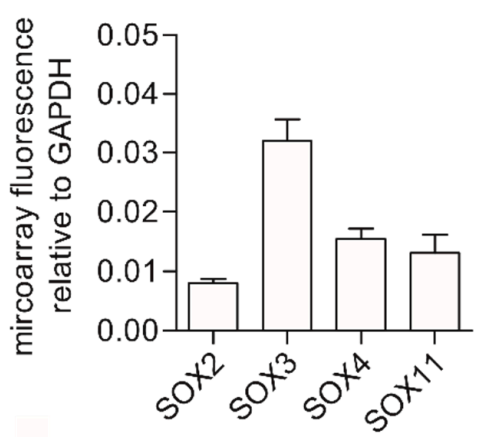

B

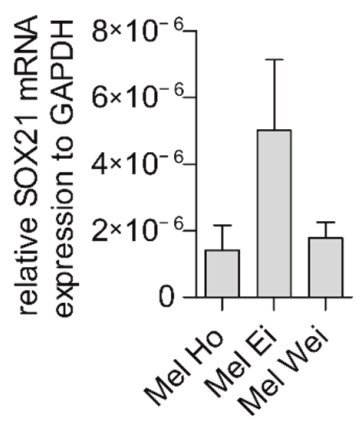

E

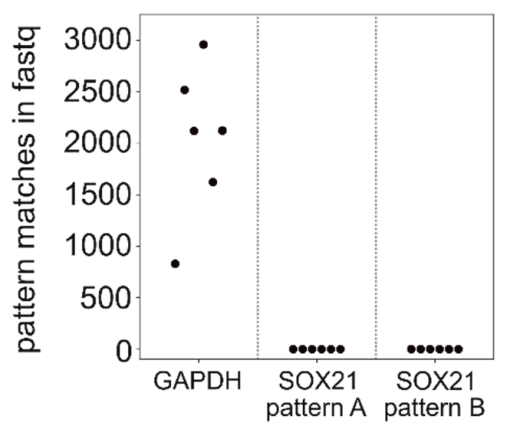

C

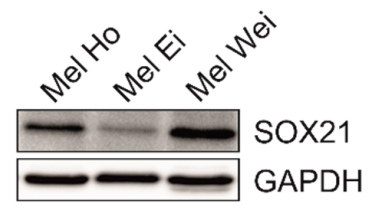

Figure 2. Comparison of cDNA microarray analysis with RNA-seq and qRT-PCR measurements for different $S O X$ genes. (A) Evaluation of the raw data from transcriptome analysis with a cDNA microarray (green, Supplementary Table S4) and RNA-seq (blue, Supplementary Table S5) for GAPDH and SOX21. (B) Expression analysis of SOX21 mRNA with qRT-PCR in different melanoma cell lines. (C) Western blot analysis revealing the SOX21 protein levels in human melanoma cell lines. (D) Exemplary analysis of SOX21 mRNA expression in cDNA and library samples with qRT-PCR for two melanoma cell lines (Mel Juso, Mel Wei). (E) The absolute numbers of pattern matches with no mismatches are shown for the GAPDH and two SOX21 patterns for the Mel Im, Mel Wei, and Mel Juso cell lines. Both paired-end fastq files per cell line were used for the pattern search. (F) Evaluation of the microarray fluorescence signal for further SOX genes relative to GAPDH based on cDNA array data (Supplementary Table S4). (G) Representation of the normalized RNA-seq read counts for further $S O X$ genes relative to GAPDH (Supplementary Table S5). (H) Comparison of qRT-PCR measurements for further SOX genes of cDNA and library samples analogous to Figure 2D. For all analyses $(\mathbf{A}-\mathbf{H})$, at least two different melanoma cell lines were used with three biological replicates. GAPDH was used as an internal control for each transcriptome method and cell line separately. The dot and box plots show the mean \pm SEM.

To investigate whether the loss of detection was observed for the whole SOX family, we chose additional SOX genes (SOX2, SOX3, SOX4, and SOX11) and compared the cDNA 
array data (Supplementary Table S4) with the RNA-seq read counts (Supplementary Table S5) relative to GAPDH. All of the SOX genes showed a positive signal with the cDNA microarray gene expression analysis (Figure 2F). Interestingly, the RNA-seq experiments resulted in no read counts for SOX3 and SOX11, while SOX2 and SOX4 showed a comparable expression in RNA-seq to that observed in the cDNA arrays (Figure 2G). This was already visible within the comparison of the transcriptome data from the microarray and RNA-seq analysis (Figure 1A,B, red label) and was further confirmed in another transcriptome analysis dataset (Supplementary Figure S1B,C). We additionally quantified the mRNA levels of these SOX genes with qRT-PCR from cDNA in comparison with prepared library samples (Figure $2 \mathrm{H}$ ) and observed the same aforementioned loss in our RNA-seq library samples. We were able to detect fluorescence signals for all of the investigated $S O X$ genes through the microarray analysis, and we were able to quantify the cDNA expression levels; however, no RNA-seq reads were found for SOX3 or SOX11, and no amplification was observed when analyzing the respective library samples with qRT-PCR. We also compared the cDNA array (Supplementary Figure S2A) and the RNA-seq data for the entire SOX gene family (Supplementary Figure S2B) and demonstrated that there were genes in addition to SOX21, SOX3, and SOX11 within the SOX family that could not be detected with RNA-seq, but resulted in a fluorescence signal in the microarray analysis.

\subsection{Analysis of Further Genes through Transcriptome Analysis}

To rule out that the observed loss of gene information was mainly associated with SOX-transcription factors, we further analyzed YAP1 (yes-associated protein 1) and TAZ (transcriptional co-activator with PDZ-binding motif) within the datasets. Therefore, we analyzed the fluorescence signals detected with the microarray analysis for YAP1 and TAZ (Figure 3A) and the read counts measured with RNA-seq for both genes (Figure 3B). We were able to detect both genes with both methods (Figure $3 \mathrm{~A}, \mathrm{~B})$. These results were again independent of the housekeeper and were demonstrated in parallel for ACTG1 within two different datasets of transcriptome analyses for each method (Supplementary Figure S1D,E). The microarray data indicate that genes that have generally lower detection signals (Figure 3A and Figure S1D) are potentially more difficult to detect with RNA-seq (Figure $3 \mathrm{~B}$ and Figure S1E).

In addition, the effect of genes that are undetectable or difficult to detect with RNA-seq was not only observed for transcription factors, as shown in Figure 1A,B (black dots: n.d. RNA-seq) and the corresponding Supplementary Table S3, but also for S100A7, which is known in the literature to be expressed in melanoma, as well as chemokine 5 (CXCL5), just to name a few [18-20].

\subsection{Discussion of Possible Causes of Gene Loss}

After demonstrating the loss of information by comparing the results of RNA-seq, microarrays, and qRT-PCR based on various examples and independent of the cell line and housekeeper, we aimed to understand the underlying causes for why certain genes are more difficult to detect or are not detectable with RNA-seq. First, we focused on the GC content, which could make it challenging to identify the genes investigated in this study. Price A. et al. (2017) discussed this before and observed a relationship between a varying GC content, local RNA secondary structure, and read depth [21]. However, linking the GC content to the detection of the genes of interest did not lead to conclusive results. We could not see any dependence of the GC content on the detectable or undetectable genes with RNA-seq (Supplementary Table S6). Because RNA directly reaches its folded state after synthesis, we further focused on the secondary structure of the RNA [22]. Here, we used a thermodynamic structure prediction tool to predict the minimum free-energy structures and base-pair probabilities from single RNA sequences according to the Zuker algorithm [23]. We calculated the total of the quotient out of the minimum free energy of the mRNA secondary structure and the number of nucleotides of each RNA sequence to adjust to the mRNA length (Supplementary Table S6). These additional analyses showed 
a lower quotient for detectable genes (Figure 3C, blue dots) and a higher quotient for undetectable genes (Figure $3 \mathrm{C}$, red dots). This led to the assumption that the secondary structure could be one of the main criteria for the accessibility of the RNA for further processing steps and, consequently, for a successful RNA sequencing approach.

A

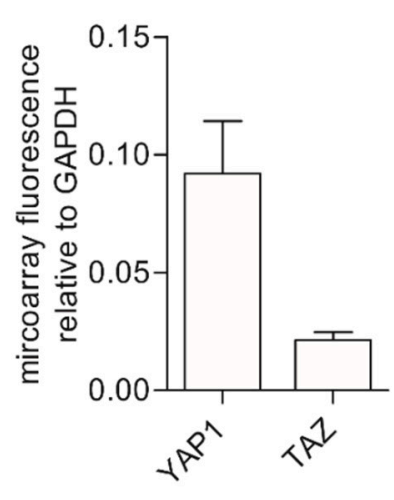

D

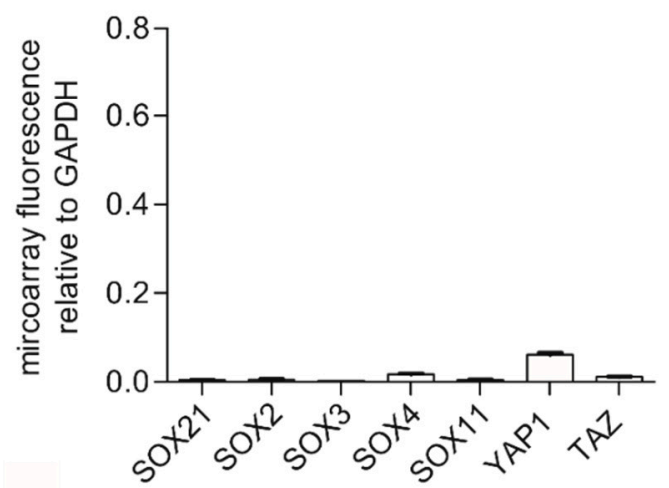

B

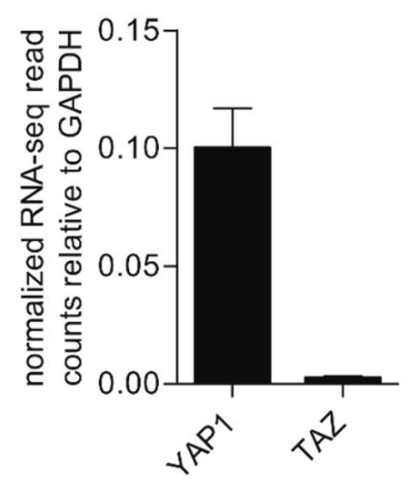

C

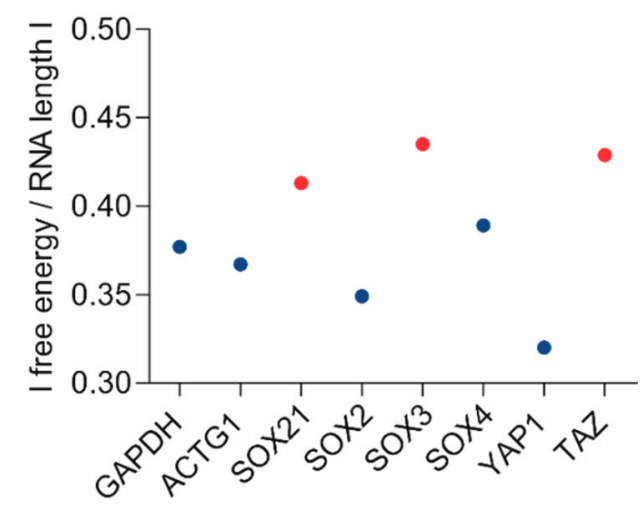

E

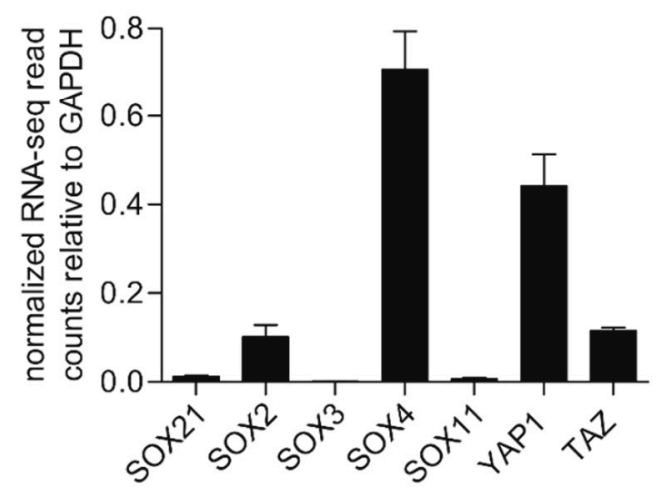

Figure 3. Detection of genes using transcriptome analysis. (A) Analysis of the microarray fluorescence signals of $Y A P 1$ and TAZ (Supplementary Table S4) relative to GAPDH within three different cell lines. (B) YAP1 and TAZ detection with RNA-seq (Supplementary Table S5) normalized for each cell line to GAPDH. (C) Trend for genes that are detectable by using RNA-seq (blue dots) as a function of the |free energy| divided by the RNA length. Undetectable genes via RNA-seq are illustrated as red dots. (D) Analysis of the microarray data from Hoek et al. (GSE4845 GPL570) for some genes of interest. (E) Analysis of RNA-seq datasets of mechanically fragmented RNA samples (SOX3 mean value: $0.88 \times 10^{-3}$ ) from Kunz et al. (GSE112509). GAPDH was used for normalization. The box plots show the mean $\pm \mathrm{SEM}$.

For the processing, library preparation, and subsequent sequencing, RNA molecules must initially be sheared into smaller pieces to be compatible with most deep sequencing technologies, such as RNA-seq [1]. There are two options for RNA shearing: chemical and mechanical $[7,24]$. Chemical shearing includes shearing with enzymes (RNase III), alkaline solutions, or divalent cations $\left(\mathrm{Mg}^{++}, \mathrm{Zn}^{++}\right)$with incubation at an elevated temperature (70 to $95^{\circ} \mathrm{C}$ ), while mechanical shearing comprises acoustic shearing (nebulization, sonification) $[7,24,25]$. It is possible that different processing methods are differently affected by the RNA secondary structure [7].

\subsection{Analysis of Mechanically Sheared RNA-Seq Datasets}

To exclude that the described observations only apply to our datasets, we analyzed already published RNA-seq data from Kunz et al. (accessible NCBI Gene Expression Omnibus (GEO), GEO Series GSE112509, melanoma tissue) and the cDNA microarray data from Hoek et al. (GEO Series accession GSE4845 GPL570, melanoma cell lines) [26,27], 
keeping in mind that comparing expression data obtained in different laboratories and using different biological materials can also yield discrepancies. Differently from our enzymatic shearing approach, the samples of Kunz et al. were preprocessed through mechanical shearing [26]. Due to the fact that Kunz et al. used melanoma tissue in an independent study, this comparison with our datasets only gives an approximation of the difference between the two fragmentation methods. Interestingly, the published analysis results of both the microarray (Figure 3D, Hoek et al. [27]) and RNA-seq (Figure 3E, Kunz et al. [26]) showed a different output concerning the genes of interest compared to the data analysis of our RNA-seq approach. Here, the expression of all genes of interest (SOX2, SOX3, SOX4, SOX11, SOX21, YAP1, and TAZ) could be determined with RNA-seq. We further investigated $Y A P 1$ and TAZ and, in comparison with our data, were able to detect both genes through mechanical RNA sample processing (Figure 3E). We further analyzed the whole SOX gene family for the microarray analysis and RNA-seq of the datasets from Hoek et al. (Supplementary Figure S2C) and Kunz et al. (Supplementary Figure S2D), respectively $[26,27]$. By comparing the RNA-seq detection of the SOX-gene family with different fragmentation methods, it became clear that several genes remained measurable during mechanical processing (Supplementary Figure S2D) compared to chemical fragmentation (Supplementary Figure S2B). Therefore, it seems that chemical shearing is less effective for highly structured RNA, and there are differences within the outputs of RNA-seq with different fragmentation methods. However, this assumption remains to be verified for melanoma cell lines that are preprocessed through mechanical shearing, as the effects of other factors (such as the different biological materials used) must be kept in mind.

Consequently, as described by Griffith M. et al., the RNA-seq method is still an area under development, and changing experimental design parameters can have significant impacts on the strategy of the analysis and on the results [28]. This is also demonstrated by the possibility of using different kits from various suppliers for sample/library preparation. In summary, when using different datasets generated by different groups (e.g., provided on NCBI GEO), it is important to pay attention to the sample/library preparation and data generation, as well as to confirm the results with another method-e.g., qRT-PCR.

\section{Materials and Methods}

\subsection{Cell Lines and Culture Conditions}

Human melanoma cell lines (501 Mel, A375, Mel Ei, Mel Ho, Mel Im, Mel Juso, and $\mathrm{Mel} \mathrm{Wei}$ ) were described previously [29-31]. The human cell lines $\mathrm{Mel} \mathrm{Ei,} \mathrm{Mel} \mathrm{Ho}, \mathrm{Mel} \mathrm{Im}$, $\mathrm{Mel}$ Juso, and Mel Wei were provided by Dr. Judith Johnson (LMU, Munich, Germany). A375 cells (CRL-1619) were obtained from ATCC and $501 \mathrm{Mel}$ cells were provided by Dr. Ruth Halaban (Department of Dermatology, Yale University School of Medicine, New Haven, CT, USA). Cells were maintained in Dulbecco's modified Eagle's medium (DMEM) supplemented with penicillin (400 units $\left.\mathrm{mL}^{-1}\right)$, streptomycin $\left(50 \mathrm{mg} \cdot \mathrm{mL}^{-1}\right)$, and $10 \%$ fetal calf serum (FCS; Sigma Aldrich, St. Louis, MO, USA). For the melanoma cell lines $501 \mathrm{Mel}$, $\mathrm{Mel} \mathrm{Ho}$, and Mel Juso, Roswell Park Memorial Institute (RPMI) 1640 medium with $\mathrm{NaHCO}_{3}$ was used with the same supplements. All cell lines were split at a ratio 1:5 on every 3rd day and were incubated at $37{ }^{\circ} \mathrm{C}$ in a humidified atmosphere containing $8 \% \mathrm{CO}_{2}$. The cell lines Mel Im and Mel Juso were analyzed with both RNA-seq and a cDNA microarray (see Sections 3.4 and 3.5). WM3211, WM1366, and WM793, which were analyzed within a second dataset for RNA sequencing, were previously described and provided by Dr. Meenhard Herlyn (Wistar Institute, Philadelphia, PA, USA) [32]. These Wistar cell lines were maintained in a culture medium consisting of MCDB153 (Sigma Aldrich) with 20\% Leibovitz's L-15 (PAA Laboratories, Pasching, Austria), 2\% FCS, $1.68 \mathrm{mM} \mathrm{CaCl}_{2}$ (Sigma Aldrich), and $5 \mu \mathrm{g} \cdot \mathrm{mL}^{-1}$ insulin (Sigma Aldrich), and they were incubated at $37^{\circ} \mathrm{C}$ in an atmosphere containing $5 \% \mathrm{CO}_{2}$. 


\subsection{Protein Analysis (Western Blotting)}

Cell lysates were prepared as described; $30 \mu \mathrm{g}$ was loaded per lane, separated on a $12.75 \%$ sodium dodecyl sulphate polyacrylamide gel electrophoresis (SDS-page) gel, and blotted onto a polyvinylidene difluoride (PVDF) membrane (Bio-Rad) [33]. After blocking for $1 \mathrm{~h}$ with $5 \%$ bovine serum albumin solved in tris-buffered saline with Tween 20 (BSA/TBS-T), the membrane was incubated at $4{ }^{\circ} \mathrm{C}$ overnight with one of the following antibodies: anti-SOX21 antibody (1:2000; AMAB91311; Sigma Aldrich) or anti-GAPDH (1:1000; \#2118; Cell Signaling Technology, Danvers, MA, USA). After three steps of washing with TBS-T, the membrane was incubated for $1 \mathrm{~h}$ at room temperature with a horseradish peroxidase-coupled secondary anti-mouse or anti-rabbit antibody at 1:2000 dilution in TBS-T. After washing again, the staining was performed by using the ECL Plus Western Blotting Detection Kit (GE Healthcare Life Science Europe GmbH, Freiburg, Germany), and luminescence was measured with the Intas ECL chemocam imager.

\subsection{Analysis of Gene Expression with Quantitative Real-Time PCR (qRT-PCR)}

Isolation of total cellular RNA was performed using the E.N.Z.A. MicroElute Total RNA Kit (Omega Bio-Tek, VWR, Darmstadt, Germany) as described by the manufacturers. cDNA was generated as previously described through the use of $500 \mathrm{ng}$ RNA [34]. The description "library sample" or "library" was used to designate RNA samples that were prepared for RNA-seq analysis according to the manufacturer's instructions (Illumina, Inc., San Diego, CA, USA); they contained ligated adapters and were enriched through PCR. Therefore, $1 \mu \mathrm{L}$ out of a 1:10 dilution of these enriched library samples was used for analysis with qRT-PCR. The qRT-PCR analysis was performed on a LightCycler ${ }^{\circledR} 480$ II system (Roche, Rotkreuz, Switzerland) as described before, and it was performed with specific sets of primers (Table 1) [35]. GAPDH was used for normalization. For each gene analysis, the length of the LightCycler product was chosen to be nearly identical to the product length of the housekeeper GAPDH. Therefore, two different GAPDH primers were required for normalization. For all qRT-PCR analyses, at least two different melanoma cell lines were used with three biological replicates.

Table 1. Oligonucleotide sequences for the qRT-PCR analysis.

\begin{tabular}{|c|c|c|c|c|}
\hline Primer & Forward Primer $5^{\prime}-3^{\prime}$ & Reverse Primer $5^{\prime}-3^{\prime}$ & $\begin{array}{c}\text { Product Size in } \\
\text { bp }\end{array}$ & $\begin{array}{l}\text { Melting Peak } \\
\text { in }{ }^{\circ} \mathrm{C}\end{array}$ \\
\hline GAPDH & TGGGGAAGGTGAAGGTCGGA & TTGATGACAAGCTTCCCGTTC & 207 & 83 \\
\hline GAPDH & GGCTCTCCAGAACATCATCCCTGC & GGGTGTCGCTGTTGAAGTCAGAGG & 269 & 88 \\
\hline SOX21 & GGAGAACCCCAAGATGCACA & CCGGGAAGGCGAACTTGT & 202 & 89 \\
\hline SOX2 & GAACCAGCGCATGGACAGTT & AGCCGTTCATGTAGGTCTGC & 199 & 91 \\
\hline SOX3 & GATAAGCCTACCCTTCCCGC & GTGTCCCTACGGGGTTCTTG & 196 & 92 \\
\hline SOX 4 & CAGCAAACCAACAATGCCGA & GATCTGCGACCACACCATGA & 209 & 93 \\
\hline SOX11 & GAGGGCGAATTCATGGCTTG & ATTTTCCAGCGCTTGCCCAG & 199 & 89 \\
\hline YAP1 & CCCTCGTTTTGCCATGAACC & ACCATCCTGCTCCAGTGTTG & 286 & 88 \\
\hline TAZ & TGGACCAAGTACATGAACCACC & AAATTCTGCTCCTCGGCACA & 278 & 88 \\
\hline
\end{tabular}

\subsection{Transcriptome Analysis with cDNA Microarrays}

After the cellular RNA was isolated, sample processing was performed at an Affymetrix Service Provider and Core Facility, “KFB-Center of Excellence for Fluorescence Bioanalytics" (Regensburg, Germany; www.kfb-regensburg.de (accessed on 8 December 2020)). Samples were generated according to the manufacturer's instructions for the Affymetrix GeneChip WT Plus reagent kit (Thermo Fisher Scientific, Waltham, MA, USA). The fluorescence signals were measured with an Affymetrix GeneChip Scanner 3000 7G and normalized with the RMA algorithm. In this study, two datasets were used. The first dataset contained two different cell lines ( $\mathrm{Mel} \mathrm{Im}$ and Mel Juso) that were analyzed in two biological replicates (Supplementary Table S1). The second dataset (Supplementary Table S4) contained three further melanoma cell lines (501 Mel, A375, and $\mathrm{Mel} \mathrm{Ho}$ ) in a single replicate each. 


\subsection{Transcriptome Analysis with Total RNA-Seq}

RNA-seq samples and libraries were prepared as described previously [36]. Library preparation was performed with at least two biological replicates. The resulting libraries were checked for size (200-500 bp) and concentration by Tape Station 4200 (Agilent) using the High-Sensitivity DNA Kit (Agilent). Qualified RNA-seq libraries were sequenced according to the $75 \mathrm{bp}$ paired-end RNA-seq approach on a HiSeq3000/4000 (Illumina, Inc.) with an average number of 20 million reads per sample. Paired-end reads were aligned to the human reference genome (hg38) and processed as described previously [37]. The resulting annotated reads normalized to library size were used for further analysis. For analysis within this manuscript, two different datasets were used. The first dataset (Supplementary Table S2) contained the same cell lines ( Mel Im and Mel Juso) analyzed with a cDNA microarray. The second dataset (Supplementary Table S5) contained three additional melanoma cell lines (WM3211, WM1366, and WM793). In both datasets, the cell lines were analyzed in two biological replicates.

\subsection{Analysis of the RNA}

The free energy of the RNA secondary structure was analyzed with the RNAfold server of the ViennaRNA Web service (http:/ / rna.tbi.univie.ac.at/ (accessed on 2 March 2021)). This tool calculates the minimum free energy of an RNA sequence based on the Zuker algorithm [23]. For calculations of the GC content of RNA molecules, the online tool endmemo (http://www.endmemo.com/bio/gc.php (accessed on 11 March 2021)) was used.

\subsection{Statistical Analysis}

The results are shown as the mean \pm SEM (standard error of the mean) calculated with the GraphPad Prism software (GraphPad Software, Inc., San Diego, CA, USA). A correlation analysis was performed using R v.4.0.3 with the help of the ggplot2 and ggpubr v.0.4.0 packages by A. Kassambara (https:/ / github.com/kassambara / (accessed on 8 December 2020)) [38-40].

\subsection{Accession Numbers}

The data were deposited in the NCBI Gene Expression Omnibus under GEO:

Kunz et al. [26]; GSE112509; DESeq2_normalized_counts file: (Ensemble-ID: ENSG0000 0111640 = GAPDH; ENSG00000125285 = SOX21); https: / /www.ncbi.nlm.nih.gov / geo / query / acc.cgi?acc $=$ GSE112509 (accessed on 11 January 2021).

Hoek et al. [27]; GSE4845-GPL570_series_matrix: (ID_REF: 212581_x_at = GAPDH; 208468_at = SOX21); https: / /www.ncbi.nlm.nih.gov /geo/query /acc.cgi?acc=GSE4845 (accessed on 11 January 2021).

\section{Conclusions}

This study indicates that genes with strong secondary-structured mRNA are difficult to determine in RNA sequencing after chemical shearing. Chemical shearing seems to fail in breaking up strong secondary RNA structures. However, we cannot state whether the method of chemical shearing also has advantages with regards to the detection of other genes. Therefore, we strongly suggest that, as for cDNA array analyses at the time that that method was established, all pros and cons must be defined in detail and made public; if possible, they also need to be incorporated into bioinformatical analyses. Although RNA-seq offers immense advantages over microarray analysis, there are challenges that need to be addressed. Therefore, we recommend validating the results of transcriptome analyses by using at least one additional method.

Supplementary Materials: The following are available online at https:/ /www.mdpi.com/article/10 .3390/ijms22179349/s1. 
Author Contributions: Conceptualization, A.K.B., M.K.-F., N.R. and S.F.; methodology, all authors; software, M.K.-F., S.F. and N.R.; validation, M.K.-F., S.F. and N.R. with contributions from all authors; formal analysis, A.K.B., M.K.-F., S.F. and N.R.; investigation, A.K.B., M.K.-F. and N.R.; resources, A.K.B. and M.K.-F.; data curation, A.K.B. and M.K.-F.; writing-original draft preparation, N.R., A.K.B., M.K.-F., S.F. and S.K.; writing-review and editing, all authors; visualization, N.R., S.F. and M.K.-F.; supervision and project administration, A.K.B.; funding acquisition, A.K.B. and M.K.-F. All authors have read and agreed to the published version of the manuscript.

Funding: The work was funded by the Deutsche Forschungsgemeinschaft (DFG, German Research Foundation), research group FOR 2127.

Institutional Review Board Statement: Not applicable.

Informed Consent Statement: Not applicable.

Data Availability Statement: External data sources used in this study are cited in the article. The extracted data are available in the Supplementary Material.

Acknowledgments: We thank the biomedical sequencing facility (BSF) of the CeMM (Vienna; Austria), the KFB (Kompetenzzentrum Fluoreszente Bioanalytik; Regensburg; Germany) and the Genomics Platform of the Regensburg Center for Interventional Immunology (RCI, University Regensburg and University Medical Center Regensburg, Germany) for sequencing and for their help with NGS library preparation. Additionally, we thank Alexander O. Matthies (Institute of Biochemistry, Friedrich-Alexander University Erlangen-Nürnberg (FAU), Erlangen, Germany) for the technical support and Phillipp Torkler (Faculty of Computer Science, Deggendorf Institute of Technology, Deggendorf, Germany) for the help with the pattern search and fastq analysis.

Conflicts of Interest: The authors declare that the manuscript presents original research, has not been previously published, and is not being considered for publication elsewhere. The authors declare that there are no conflicts of interest. The funders had no role in the design of the study; in the collection, analyses, or interpretation of data; in the writing of the manuscript, or in the decision to publish the results.

\section{References}

1. Wang, Z.; Gerstein, M.; Snyder, M. RNA-Seq: A revolutionary tool for transcriptomics. Nat. Rev. Genet. 2009, 10, 57-63. [CrossRef] [PubMed]

2. Van Der Kloet, F.M.; Buurmans, J.; Jonker, M.J.; Smilde, A.K.; Westerhuis, J.A. Increased comparability between RNA-Seq and microarray data by utilization of gene sets. PLoS Comput. Biol. 2020, 16, e1008295. [CrossRef] [PubMed]

3. Chatterjee, A.; Ahn, A.; Rodger, E.J.; Stockwell, P.A.; Eccles, M.R. A Guide for Designing and Analyzing RNA-Seq Data. In Methods in Molecular Biology; Humana Press Inc.: New York, NY, USA, 2018; Volume 1783, pp. 35-80. [CrossRef]

4. Sayani, A.; Bueno-De-Mesquita, J.M.; Van De Vijver, M.J. Technology Insight: Tuning into the genetic orchestra using microarrayslimitations of DNA microarrays in clinical practice. Nat. Clin. Pr. Oncol. 2006, 3, 501-516. [CrossRef]

5. Murphy, D. Gene Expression Studies Using Microarrays: Principles, Problems, and Prospects. Adv. Physiol. Educ. 2002, 26, 256-270. [CrossRef] [PubMed]

6. Hung, J.H.; Weng, Z. Analysis of Microarray and RNA-Seq Expression Profiling Data. Cold Spring Harb. Protoc. 2017, 3, 191-196. [CrossRef]

7. Hrdlickova, R.; Toloue, M.; Tian, B. RNA-Seq methods for transcriptome analysis. Wiley Interdiscip. Rev. RNA 2017, 8, e1364. [CrossRef]

8. $\quad$ Everaert, C.; Luypaert, M.; Maag, J.L.V.; Cheng, Q.X.; Dinger, M.E.; Hellemans, J.; Mestdagh, P. Benchmarking of RNA-sequencing analysis workflows using whole-transcriptome RT-qPCR expression data. Sci. Rep. 2017, 7, 1559. [CrossRef]

9. Shahjaman, M.; Manir Hossain Mollah, M.; Rahman, R.; Islam, S.S.; Mollah, N.H. Robust identification of differentially expressed genes from RNA-seq data. Genomics 2019, 112, 2000-2010. [CrossRef]

10. Stark, R.; Grzelak, M.; Hadfield, J. RNA sequencing: The teenage years. Nat. Rev. Genet. 2019, 20, 631-656. [CrossRef]

11. Murdock, D.R. Enhancing Diagnosis Through RNA Sequencing. Clin. Lab. Med. 2020, 40, 113-119. [CrossRef]

12. Podnar, J.; Deiderick, H.; Huerta, G.; Hunicke-Smith, S. Next-Generation Sequencing RNA-Seq Library Construction. Curr. Protoc. Mol. Biol. 2014, 106, 4.21.1-4.21.19. [CrossRef]

13. Wang, C.; Gong, B.; Bushel, P.R.; Thierry-Mieg, J.; Thierry-Mieg, D.; Xu, J.; Fang, H.; Hong, H.; Shen, J.; Su, Z.; et al. The concordance between RNA-seq and microarray data depends on chemical treatment and transcript abundance. Nat. Biotechnol. 2014, 32, 926-932. [CrossRef]

14. Su, Z.; Łabaj, P.P.; Li, S.; Thierry-Mieg, J.; Thierry-Mieg, D.; Shi, W.; Wang, C.; Schroth, G.P.; Setterquist, R.A.; Thompson, J.F.; et al. A Comprehensive Assessment of RNA-Seq Accuracy, Reproducibility and Information Content by the Sequencing Quality Control Consortium. Nat. Biotechnol. 2014, 32, 903-914. [CrossRef] 
15. Fu, X.; Fu, N.; Guo, S.; Yan, Z.; Yixue, L.; Hu, H.; Menzel, C.; Chen, W.; Philipp, K.; Zeng, R.; et al. Estimating accuracy of RNA-Seq and microarrays with proteomics. BMC Genom. 2009, 10, 161. [CrossRef]

16. Zhao, S.; Fung-Leung, W.-P.; Bittner, A.; Ngo, K.; Liu, X. Comparison of RNA-Seq and Microarray in Transcriptome Profiling of Activated T Cells. PLoS ONE 2014, 9, e78644. [CrossRef]

17. Dobin, A.; Gingeras, T.R. Optimizing RNA-Seq Mapping with STAR. In Methods in Molecular Biology; Humana Press Inc.: New York, NY, USA, 2016; Volume 1415, pp. 245-262. [CrossRef]

18. Xiong, T.-F.; Pan, F.-Q.; Li, D. Expression and clinical significance of S100 family genes in patients with melanoma. Melanoma Res. 2019, 29, 23-29. [CrossRef] [PubMed]

19. Bhalla, S.; Kaur, H.; Dhall, A.; Raghava, G.P.S. Prediction and Analysis of Skin Cancer Progression using Genomics Profiles of Patients. Sci. Rep. 2019, 9, 15790. [CrossRef]

20. Soler-Cardona, A.; Forsthuber, A.; Lipp, K.; Ebersberger, S.; Heinz, M.; Schossleitner, K.; Buchberger, E.; Gröger, M.; Petzelbauer, P.; Hoeller, C.; et al. CXCL5 Facilitates Melanoma Cell-Neutrophil Interaction and Lymph Node Metastasis. J. Investig. Dermatol. 2018, 138, 1627-1635. [CrossRef]

21. Price, A.; Garhyan, J.; Gibas, C. The impact of RNA secondary structure on read start locations on the Illumina sequencing platform. PLoS ONE 2017, 12, e0173023. [CrossRef] [PubMed]

22. Onoa, B.; Tinoco, I. RNA folding and unfolding. Curr. Opin. Struct. Biol. 2004, 14, 374-379. [CrossRef] [PubMed]

23. Zuker, M.; Stiegler, P. Optimal computer folding of large RNA sequences using thermodynamics and auxiliary information. Nucleic Acids Res. 1981, 9, 133-148. [CrossRef]

24. Roberts, A.; Trapnell, C.; Donaghey, J.; Rinn, J.L.; Pachter, L. Improving RNA-Seq expression estimates by correcting for fragment bias. Genome Biol. 2011, 12, R22. [CrossRef]

25. Mayer, A.; Churchman, L.S. Genome-wide profiling of RNA polymerase transcription at nucleotide resolution in human cells with native elongating transcript sequencing. Nat. Protoc. 2016, 11, 813-833. [CrossRef] [PubMed]

26. Kunz, M.; Löffler-Wirth, H.; Dannemann, M.; Willscher, E.; Doose, G.; Kelso, J.; Kottek, T.; Nickel, B.; Hopp, L.; Landsberg, J.; et al. RNA-seq analysis identifies different transcriptomic types and developmental trajectories of primary melanomas. Oncogene 2018, 37, 6136-6151. [CrossRef] [PubMed]

27. Hoek, K.S.; Schlegel, N.C.; Brafford, P.; Sucker, A.; Ugurel, S.; Kumar, R.; Weber, B.L.; Nathanson, K.; Phillips, D.J.; Herlyn, M.; et al. Metastatic potential of melanomas defined by specific gene expression profiles with no BRAF signature. Pigment. Cell Res. 2006, 19, 290-302. [CrossRef]

28. Griffith, M.; Walker, J.R.; Spies, N.C.; Ainscough, B.J.; Griffith, O.L. Informatics for RNA Sequencing: A Web Resource for Analysis on the Cloud. PLoS Comput. Biol. 2015, 11, e1004393. [CrossRef] [PubMed]

29. Jacob, K.; Wach, F.; Holzapfel, U.; Hein, R.; Lengyel, E.; Buettner, R.; Bosserhoff, A.-K. In vitro modulation of human melanoma cell invasion and proliferation by all-frans-retinoic acid. Melanoma Res. 1998, 8, 211-219. [CrossRef]

30. Mueller, D.W.; Bosserhoff, A. MicroRNA miR-196a controls melanoma-associated genes by regulating HOX-C8 expression. Int. J. Cancer 2011, 129, 1064-1074. [CrossRef]

31. Arnold, J.; Engelmann, J.C.; Schneider, N.; Bosserhoff, A.K.; Kuphal, S. miR-488-5p and its role in melanoma. Exp. Mol. Pathol. 2020, 112, 104348. [CrossRef] [PubMed]

32. Kappelmann-Fenzl, M.; Gebhard, C.; Matthies, A.O.; Kuphal, S.; Rehli, M.; Bosserhoff, A.K. C-Jun drives melanoma progression in PTEN wild type melanoma cells. Cell Death Dis. 2019, 10, 584. [CrossRef] [PubMed]

33. Böhme, I.; Bosserhoff, A. Extracellular acidosis triggers a senescence-like phenotype in human melanoma cells. Pigment. Cell Melanoma Res. 2020, 33, 41-51. [CrossRef]

34. Schiffner, S.; Braunger, B.M.; de Jel, M.M.; Coupland, S.; Tamm, E.; Bosserhoff, A.K. Tg(Grm1) transgenic mice: A murine model that mimics spontaneous uveal melanoma in humans? Exp. Eye Res. 2014, 127, 59-68. [CrossRef]

35. Kappelmann, M.; Kuphal, S.; Meister, G.; Vardimon, L.; Bosserhoff, A. MicroRNA miR-125b controls melanoma progression by direct regulation of c-Jun protein expression. Oncogene 2013, 32, 2984-2991. [CrossRef] [PubMed]

36. Andersson, R.; The FANTOM Consortium; Gebhard, C.; Miguel-Escalada, I.; Hoof, I.; Bornholdt, J.; Boyd, M.; Chen, Y.; Zhao, X.; Schmidl, C.; et al. An atlas of active enhancers across human cell types and tissues. Nature 2014, 507, 455-461. [CrossRef] [PubMed]

37. Kappelmann-Fenzl, M.; Schmidt, S.K.; Fischer, S.; Schmid, R.; Lämmerhirt, L.; Fischer, L.; Schrüfer, S.; Thievessen, I.; Schubert, D.W.; Matthies, A.; et al. Molecular Changes Induced in Melanoma by Cell Culturing in 3D Alginate Hydrogels. Cancers 2021, 13, 4111. [CrossRef]

38. R Core Team. R: A Language and Environment for Statistical Computing. R Found. Stat. Comput. 2020, 2. Available online: http:/ / www.r-project.org/ (accessed on 8 December 2020).

39. RStudio Team. Integrated Development for R. In RStudio; RStudio PBC: Boston, MA, USA, 2020; p. 14. Available online: https:/ / rstudio.com (accessed on 8 December 2020).

40. Wickham, H. Ggplot2: Elegant Graphic for Data Analysis; Springer: New York, NY, USA, 2016; Available online: https:/ /ggplot2 .tidyverse.org (accessed on 8 December 2020). 